\title{
The medicine distribution scheme to eradicate Ebola
}

\author{
Fei Lu \\ North China Electric Power University (Baoding), Baoding, 071000, China
}

Keywords: Ebola; cellular automata; equally distributing plan.

\begin{abstract}
The spreading of infectious diseases is a process of evolution with time going. In this paper we set up a model to simulate the dynamic spreading process of the virus basing on the general model: cellular automata model. Through the improved cellular automata model, this paper finally provides an optimal decision for distributing medical supplies.
\end{abstract}

\section{Introduction}

The existing data indicate Ebola virus quickly spreading in the Africa in a short time. The historical data show 602 people were infected totally in Sudan and Janbu. What is worse is that 431 of the infected persons were dead. ${ }^{[1]}$ Thus through collecting the data about the damage caused by Ebola, this paper set up a mathematic model to describe the process of it's spreading in a population. On the base of the spreading model we set up, this paper advises how to distribute the medicines reasonably to reduce the infected rate and the death rate in a region.

\section{The spreading model basing on the cellular automata [2]}

A cellular automaton is a commonly used method to describe the complicated phenomenon in reality. It is a dynamical system discrete in time, space and state so it can evolve to a complex state. Through adding some regulations set according to the rules of spreading to simulate the complicated process of spreading ${ }^{[3]}$. Based on the existing cellular automaton model, in order to simulate people's mobility problems in reality, this paper adds a random moving model obeying Poisson distribution. In order to simulate the influence of the transportation factors on the spreading of epidemics in reality, this paper applies some state variables to illustrate the terrain condition in reality in innovation. Through formulating cellular automaton evolution rules, this paper simulates the spread of epidemics. The simulated result can well fit the real condition.

\section{Generalized equal distribution model}

\subsection{Multiple objective model of distribution system [4]}

Ebola virus has spread in many places, and different locations of different countries have different demand for medical supplies. Therefore, this is a multiple objective problem concerning distribution of medical supplies. Multiple objective model of distribution involves the site selection of transfer station, the transfer path and combination of medications. We are aimed to reduce the infected rate and death rate of infected without regard to the cost involves in the distribute process. This paper creatively puts forward the generalized equal distribution model. In this model, we deliver medical supplies proportionally to different regions, and different amount corresponding to different allocation. There are four types of distribution plan:

A. distributes medical supplies according the total population in different regions;

B. distributes medical supplies according the number of infected people in different regions;

C. distributes medical supplies according the number of isolated people in different regions;

D. distributes medical supplies according the number of people dead of Ebola virus in different regions. 
When the four different distribution plans is carried out, the cellular status of the next time step update depends on the cellular status of previous time step, which will make the process of distribution become intuitive, simple and effective.

\subsection{Assumptions}

According to the methods to analyze the epidemic diseases, we apply a randomly moving cellular automaton in 2 dimensions ${ }^{[5]}$ to set up the epidemic diseases model. Combining the truly data with numerical results of a single region and a multiple region, we adopt the multiple region model for deeper research.This paper make following assumptions:

Assuming that the demand of medicine exceeds production. What's more the level of production can not increase in a short time.

At the beginning of the production of medicine, the supply capacity can be described as shown below

$F=\mu \times \boldsymbol{H}$

Where $\mu=0.5$ is the proportionality coefficient; $H$ is the maximum of infected people.

Assuming that areas demanding of medicine are widely spread on the earth;

Assuming that medicine is needed as long as Ebola virus appear;

Assuming that the demand of medicine is continuous, and interruption of medicine can make the infected situation back to the initial state;

Assuming that distribution of medicine can be done in a short time;

\subsection{The regulation in distribution of medicine}

The research of the four plans is based on different regions.

A. Distribute medical supplies according the total population:

The amount of distributed medicine can be described as shown below:

$$
Y_{i}=F \times W_{i} / \sum_{i=1}^{N} W_{i}
$$

Where, $W_{i}(\mathrm{i}=1,2, \ldots \mathrm{N})$ is the population in region numbered $\mathrm{i}$.

B. Distribute medical supplies according the number of infected people

The amount of distributed medicine can be described as shown below:

$$
Y_{i}=F \times R_{i} / \sum_{i=1}^{N} R_{i}
$$

Where, $R_{i}(\mathrm{i}=1,2, \ldots \mathrm{N})$ is the number of infected people in region numbered $i$.

C. Distribute medical supplies according the number of isolated people

The amount of distributed medicine can be described as shown below:

$$
Y_{i}=G_{i} / \sum_{i=1}^{N} G_{i}
$$

Where, $G_{i}(\mathrm{i}=1,2, \ldots \mathrm{N})$ is the number of isolated people in region numbered $i$.

D. Distribute medical supplies according the number of people dead of Ebola virus

The amount of distributed medicine can be described as shown below:

$$
Y_{i}=E_{i} / \sum_{i=1}^{N} E_{i}
$$

Where, $E_{i}(\mathrm{i}=1,2, \ldots \mathrm{N})$ is the number of people dead of Ebola virus in region numbered $i$.

\subsection{Solution and Result}

According to the methods to analyze the epidemic diseases, we apply a randomly moving cellular automaton in 2 dimensions ${ }^{[7]}$ to set up the epidemic diseases model. The cure rate of infected people who is isolated is updated according to the four different distribution plans. Results of four different distribution plans are shown in the following four figures. 


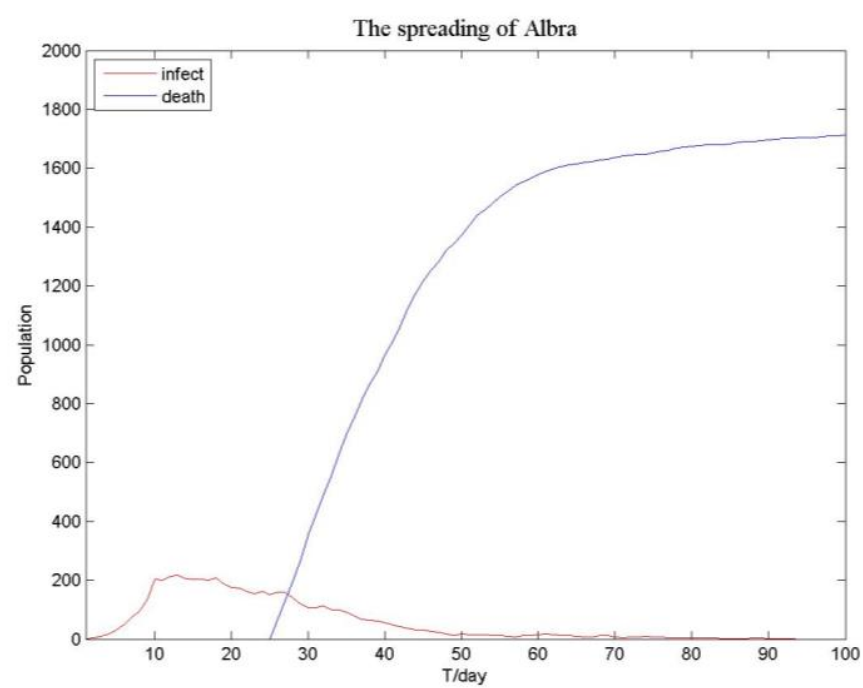

Figure 1 Infected people and dead people in plan A

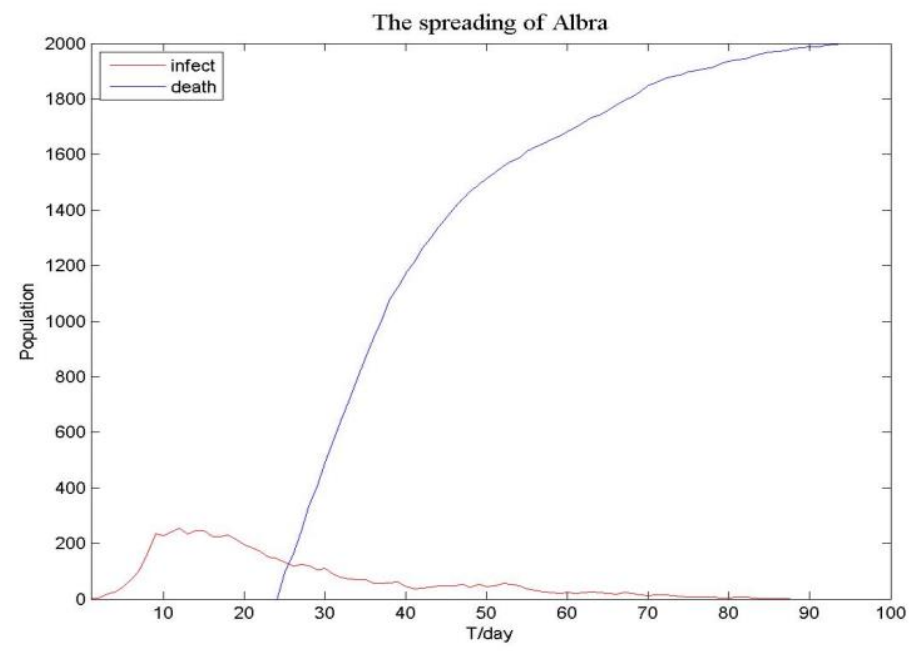

Figure 2 Infected people and dead people in plan B

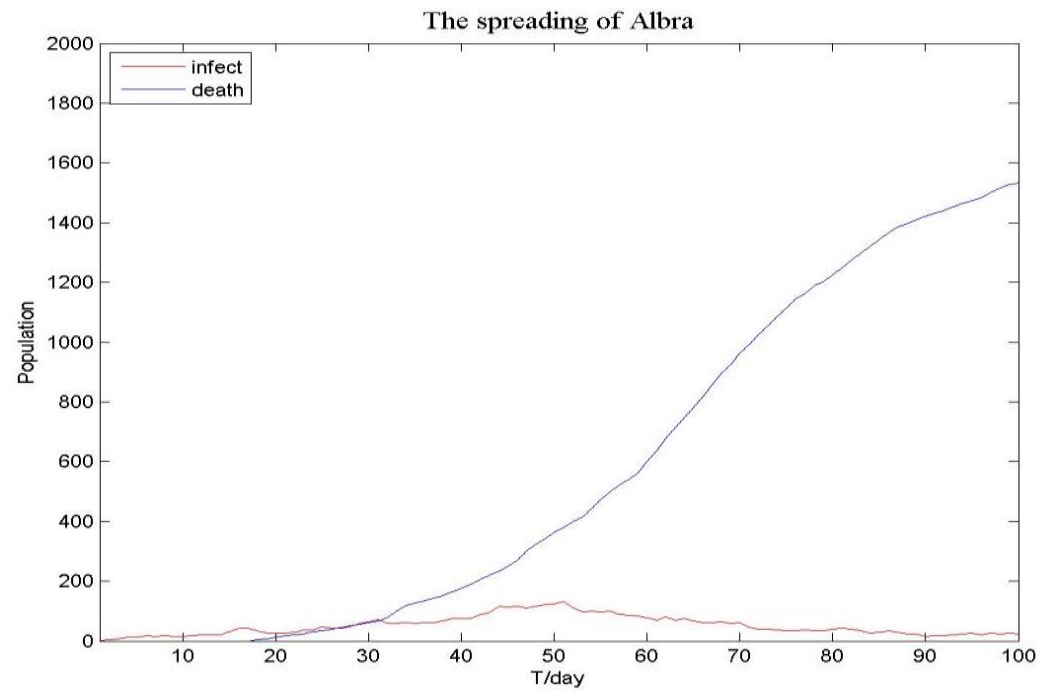

Figure 3 Infected people and dead people in plan C 


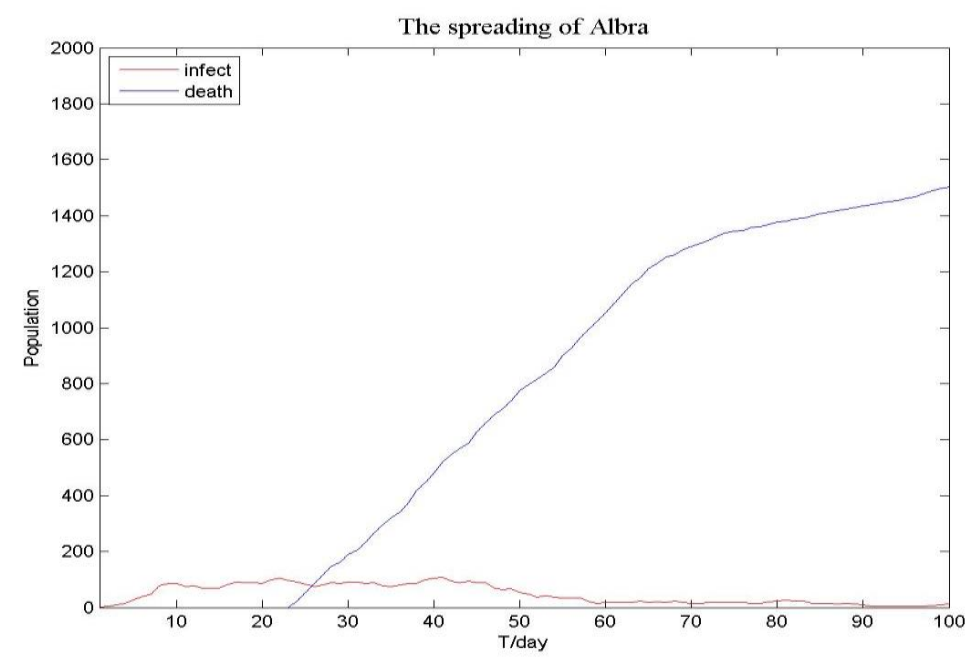

Figure 4 Infected people and dead people in plan D

\subsection{Analysis of the Result}

From figure1 and figure 2, we can see that the amount of infected people in the two different plans agree with each other very well. The number of infected people here is simulated, but we cannot know the exact value of it. The number of infected people is a certain proportion of the total number of population. Therefore, the difference between the amount of distributed medicine in the simulation and the reality is very small, so does the control effect in spreading of Ebola virus. From figure 3 and figure 4 , we can draw a conclusion that the plan in which medical supplies is distributed according the number of isolated people or the number of dead people is very effective. The amounts of infected people in the two different plans agree with each other very well and the maximum and time step differs very little. The number of isolated people is the infected people we have find. Distributing medicine according to this number is the method the government used to control large flu. The number of isolated people is a certain scale of the number of dead people. Therefore, the difference between these two methods is very small, so does the control effect in spreading of Ebola virus.

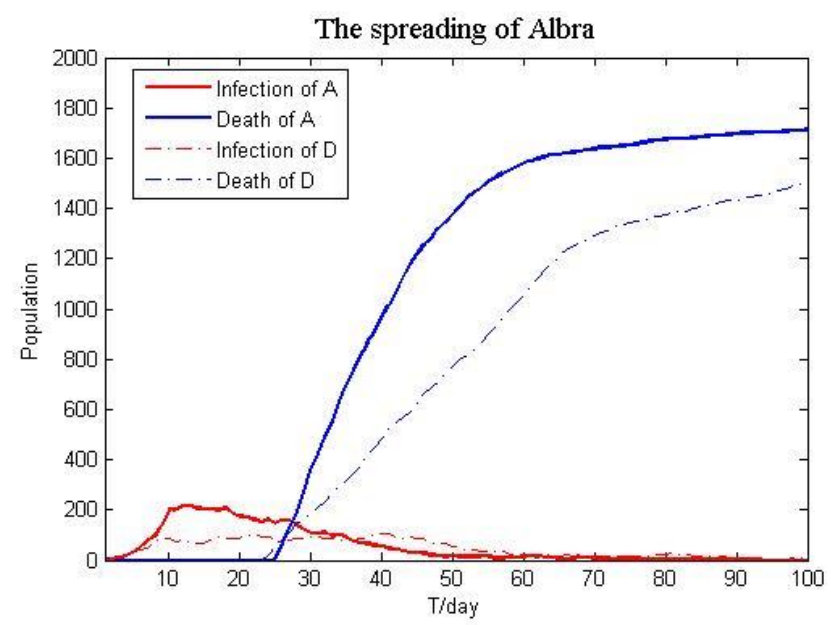

Figure 5 comparison between plan A and plan D

The results of four different distribution plans indicate that plan A and plan B have similar effect, while plan $\mathrm{C}$ and plan D have similar effect. What's more, the latter two plans are more efficient than the first two plans when control effect of Ebola virus is discussed. From figure 5, we can see that the maximum number of infected people in plan $\mathrm{A}$ is twice as what in plan $\mathrm{D}$, and the number of dead people in plan A is bigger than what in plan D. That is to say, the total number of population cannot represent the situation of Ebola virus. The medicine distribution plan should be given by considering the truly spreading of Ebola virus. The number of dead people can reflect the Ebola virus 
situation. Thus, plan D in which medicine is distributed according to the number of dead people can obtain optimal results.

\section{Summary}

Firstly, based on the existing cellular automaton model, this paper improves the model. Through formulating cellular automaton evolution rules, the result of the spread of epidemics can well fit the real condition. Secondly, we respectively distribute the vaccines basing on the population of a region, infected population, isolated population and death toll. Simulate the four distribution methods in the cellular automata model and get the simulated results. The results tell us that it is much better to distribute the vaccine basing on the death rate in a region.

\section{References}

[1]http://apps.who.int/ebola/en/ebola-situation-report/situation-reports/ebola-situation-report-4-febr uary-2015(2015.2.8)

[2] Von Neumann J.The general and logical theory of automata[C].In: Jeffries L An ed.Cerebral Mechanisms in Behavior-the Hixon Symposium, New York: Wiley, 1951

[3] Fumin Shen, Principles and methods of epidemiology [M]shanghai:Fudan University Press, 2001.

[4] Weichao Li. Under the natural disaster study on the model of multi-objective distribution of emergency supplies [D]. Huazhong University of science, 2012.

[5] Xin Yu, Xiaodong Duan, Xiangdong Liu, Based on cellular automaton epidemic model and its simulation $[\mathrm{J}]$. Computer engineering and applications, 2005. 